\title{
Improved ramped bunch train to increase the transformer ratio of a two-channel multimode dielectric wakefield accelerator
}

\author{
G. V. Sotnikov ${ }^{1,2, *}$ and T. C. Marshall ${ }^{2,3, \dagger}$ \\ ${ }^{1}$ NSC Kharkov Institute of Physics and Technology, 61108 Kharkov, Ukraine \\ ${ }^{2}$ Omega-P, Inc., New Haven, Connecticut 06511, USA \\ ${ }^{3}$ Columbia University, New York, New York 10027, USA \\ (Received 29 October 2010; published 11 March 2011)
}

\begin{abstract}
Here we show a possibility of applying the ramped drive bunch train (RBT) technique to a two-channel coaxial dielectric wakefield accelerator (CDWA). For numerical research we study a $28 \mathrm{GHz}$ structure with two nested alumina cylindrical shells having these diameters: outer shell, OD $=28.1 \mathrm{~mm}$, $\mathrm{ID}=27 \mathrm{~mm}$; inner shell, $\mathrm{OD}=6.35 \mathrm{~mm}$, ID $=4.0 \mathrm{~mm}$. The structure is to be excited by a train of four annular bunches having energy $14 \mathrm{MeV}$ and axial rms length $1 \mathrm{~mm}$; the total charge of bunches is $200 \mathrm{nC}$. In the case of equally charged drive bunches, spaced apart by the principal wakefield wavelength $10.67 \mathrm{~mm}$, we obtained transformer ratio $T=3.4$. If the bunch charge is increasing as the ratio $1: 3: 5: 7$ and the bunches are spaced by one and one-half wavelengths, we obtained $T=3.8$. We found that if the charge ratios are 1.0:2.4:3.5:5.0 and the spaces between the bunches are 2.5, 2.5, and 4.5 wakefield periods, then $T$ increases strongly, $T \sim 20$. The RBT also can be used successfully in a high gradient $\mathrm{THz}$ CDWA structure. A particle-in-cell simulation shows that the four drive bunches can move without appreciable distortion.
\end{abstract}

DOI: 10.1103/PhysRevSTAB.14.031302

PACS numbers: 41.75.Ht, 41.75.Lx, 41.75.Jv, 96.50.Pw

\section{INTRODUCTION}

Dielectric wakefield accelerator devices have become an attractive alternative to conventional metallic-structure elements used for electron/positron linear colliders [1]. In dielectric wakefield accelerator (DWA) devices, a high energy drive bunch or train of drive bunches sets up a wakefield via Cherenkov radiation; some of this energy is then transferred to a trailing bunch positioned to receive axial accelerating force. Recent interest in this concept has been rekindled by a finding that dielectrics can withstand very high fields $(>1 \mathrm{GeV} / \mathrm{m})$ for the short times involved in the passage of charged bunches along the dielectriclined channel [2]. However, other than the possibility to develop high accelerating gradients, an accelerator should have other advantages, such as an attractive transformer ratio.

Transformer ratio $(\mathbf{T})$, which is a measure of the efficacy by which energy provided by a drive bunch is transferred to a bunch that is to be accelerated in a DWA structure, is generally $<2$ in a collinear device, namely, one in which the drive bunch and the bunch to be accelerated ("witness" bunch here) travel along the same path [3-6]. The $\mathbf{T}$ is customarily defined to be the ratio of the peak accelerating

\footnotetext{
*sotnikov@kipt.kharkov.ua

${ }^{\dagger}$ tcm2@columbia.edu
}

Published by American Physical Society under the terms of the Creative Commons Attribution 3.0 License. Further distribution of this work must maintain attribution to the author(s) and the published article's title, journal citation, and DOI. field set up by a drive bunch to the average energy loss per unit charge of particles in the drive bunch $[3,4]$. On the other hand, some authors [7-10] use the ratio of the maximum energy gain of the witness bunch to the maximum energy loss of the drive bunch. There are two ways to increase the $\mathbf{T}$. The first has been to use a train of drive bunches that have a certain programmed charge and spacing determined by a simple algorithm $[7,8,10,11]$ to drive wakefields in a collinear device: this is termed a "ramped bunch train" (RBT). This method has been tried experimentally with modest success $[7,8]$, and it is most suitable when the drive bunch can excite a single mode in the device. Another technique is to separate the drive and witness bunch channels: this has been done successfully at CLIC [12] and at the Argonne Wakefield Accelerator facility $[13,14]$. Our recent effort to develop a two-channel wakefield accelerator structure that encloses both channels in one assembly [1] has found that a $\mathbf{T} \sim 5-6$ can be obtained in a coaxial DWA configuration (CDWA) in which the drive bunch is annular and is centered on the axis of a second accelerating channel that carries a witness bunch (see Fig. 1). This configuration has been found to transport a short train of drive bunches, as well as the witness bunch, satisfactorily [1]. Comparison of $\mathbf{T}$ for the ramped bunch train to that of the single drive bunch is a satisfactory indication of any improvement of $\mathbf{T}$. In this paper our attention is directed to improving the transformer ratio for the CDWA device.

An example will show how the performance of an accelerator is affected by the transformer ratio. In a certain dielectric wakefield structure, let us suppose a $14 \mathrm{MeV}$ 


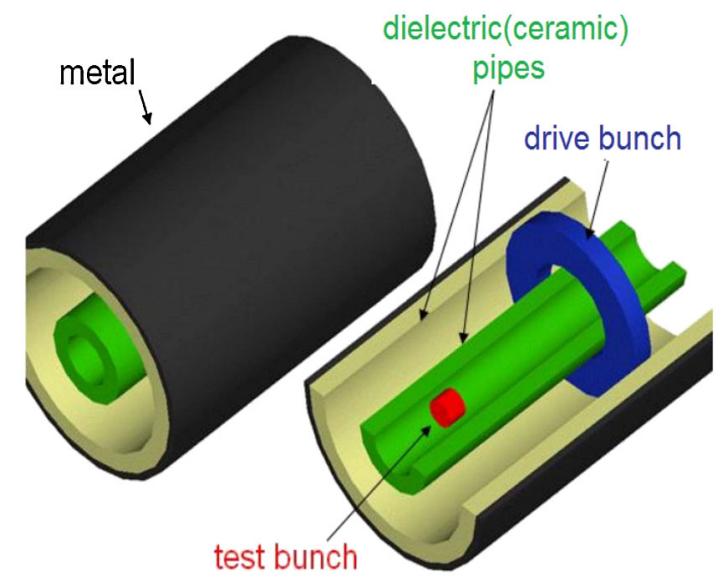

FIG. 1. Schematic of the CDWA structure, showing a single annular drive bunch followed by an accelerated witness bunch that moves along the axis.

drive bunch sets up a gradient of $\mathbf{G}=50 \mathrm{MeV} / \mathrm{m}$ for the witness bunch and $\mathbf{T}=4$. A drive bunch electron, which should then lose energy at the rate of $12.5 \mathrm{MeV} / \mathrm{m}$, can travel no more than $1.12 \mathrm{~m}$, and the witness bunch electron will gain $56 \mathrm{MeV}$. Now suppose that the gradient accelerating the witness bunch that is set up by a ramped drive bunch that has the same energy in the same structure is only $\mathbf{G}=28 \mathrm{MeV} / \mathrm{m}$, but $\mathbf{T}=20$. In that case, the drive bunch can travel $10 \mathrm{~m}$ (stability issues permitting) and the witness bunch will gain $280 \mathrm{MeV}$. (The numbers for this illustration are based on examples in the next section.) This example, which omits the influence of beam loading, illustrates the trade-off between $\mathbf{T}$ and $\mathbf{G}$ : the higher-G/lower- $\mathbf{T}$ case leads to a shorter active length, but 5 times the number of drive beam segments, while the higher-T/lower-G case implies a longer active length but fewer drive beam segments. Thus, other than high accelerating gradients, a two-beam accelerator should have a suitably high transformer ratio, so as to minimize the number of drive beam segments needed to achieve a given final test beam energy.
In the following, we find that it is possible to combine the RBT technique with the two-channel CDWA structure to improve $\mathbf{T}$. In evaluating our results in a useful form, we take the definition of $\mathbf{T}$ to be the ratio of the peak accelerating field acting on the witness bunch to the maximum energy loss of any of the drive bunches. We can compare our results for the four-bunch train with the $\mathbf{T}$ for a single bunch having the aggregate charge of all the drive bunches in the RBT. The ratios of bunch charges within the drive bunch train and the spacing between the bunches that optimize the result is specific to the structure being studied, but we find a considerable improvement in $\mathbf{T}$ can be obtained. This is potentially a useful method to optimize the design of a collider that uses CDWA modules. In what follows, we shall take bunch parameters modeled on those obtained at the AWA facility (Table I); this facility can provide a single $50 \mathrm{nC}$ bunch, but for computational purposes we assume that four $50 \mathrm{nC}$ bunches can be provided with arbitrary delays.

\section{WAKEFIELD ANALYSIS OF RAMPED DRIVE BUNCH TRAINS}

The computations were done using a particle-in-cell code, PIC SOLVER of the CST PARTICLE STUDIO being a part of the CST STUDIO SUITE 2010 bundle. Boundary conditions for PIC simulations were tangential electric fields are zero at metal surface of the waveguide and the input boundary of the unit, and output boundary is open to free space. The code computes fields, and changes in particle energy and position as the bunches move.

A factor that affects the practicality of the scheme we study is the group velocity of the Cherenkov radiation generated by the drive bunches as they enter the structure aperture [15]. Because of this factor the Cherenkov wakefield is nonzero in the region defined by the approximate inequality $\left(t-t_{0}\right) v_{g} \leq z \leq\left(t-t_{0}\right) v_{0}$, where $t_{0}$ is entry time of the first bunch in the structure, $t$ is current time, $v_{0}$ is bunch velocity, and $v_{g}$ is the group velocity of

TABLE I. Parameters used for the study of a two-channel CDWA (alumina dielectric).

\begin{tabular}{ll}
\hline \hline Design mode & $28.092 \mathrm{GHz}$ \\
External radius of outer coaxial cylinder & $14.05 \mathrm{~mm}$ \\
Inner radius of outer coaxial waveguide & $13.512 \mathrm{~mm}$ \\
External radius of inner coaxial cylinder & $3.175 \mathrm{~mm}$ \\
Accelerator channel radius (inner radius of inner coaxial cylinder) & $2.0 \mathrm{~mm}$ \\
Relative dielectric constant $\varepsilon$ & 9.8 \\
Bunch axial rms dimension $2 \sigma_{z}$ (Gaussian charge distribution) & $2.0 \mathrm{~mm}$ \\
Full bunch length used in PIC simulation & $5 \mathrm{~mm}$ \\
Outer drive bunch radius (box charge distribution) & $10.34 \mathrm{~mm}$ \\
Inner drive bunch radius & $6.34 \mathrm{~mm}$ \\
Bunch energy & $14 \mathrm{MeV}$ \\
Total bunch charge & $200 \mathrm{nC}$ \\
Number of bunches & 4 \\
\hline \hline
\end{tabular}


the resonance wave. Within this region, the envelope of a Cherenkov signal is about constant. The plane $z^{g r}=\left(t-t_{0}\right) v_{g}$ is the trailing edge of the wakefield. This edge moves behind the electron bunch at the group velocity $v_{g}$. In this plane, the fields from the number $N_{b}^{\max }=\left(t-t_{0}\right)\left(v_{0}-v_{g}\right) / \Delta z_{0}+1$ of bunches will be added [16], where $\Delta z_{0}$ is the distance between the adjacent bunches. Everything said here is true in the case of excitation of a single resonant mode in the approximation of rigid bunches. For investigation of multimode excitation with different values of group velocities of excited modes and for a self-consistent account of energy losses of bunch particles, a full numerical simulation is required.

We now turn to the computation of some examples, where we change the drive bunch train to improve $\mathbf{T}$. The first example (Fig. 2) shows the axial wakefield trailing a single $50 \mathrm{nC}$ drive bunch, measured on the axis of the unit (along which the witness bunch moves) and along a line parallel to the axis halfway between the outer and inner radii of the drive bunch annulus. Here and in the next figures, axial distance $z$ is counted starting from the injection plane of the first bunch. From this we conclude the $\mathbf{T}$ for this structure is 3.6, calculated as the ratio of maximum accelerating wakefield on the central axis of the structure (see blue curve) to the maximum decelerating wakefield at the drive bunch, $z=168 \mathrm{~mm}$, along the center line behind the drive bunch (see red curve). It is correct to infer $\mathbf{T}$ from the wakefield behind the annular drive bunch (as shown in Fig. 2) because the transverse profile of the wakefield mode amplitudes is very nearly flat [1] across the radius of the drive bunch channel; this is caused by the large relativistic factor, even for $14 \mathrm{MeV}$. One should observe also that the wakefields are much diminished for $z<100 \mathrm{~mm}$ : this shows that the superposition of wakefields is limited to a zone behind the drive bunch depending

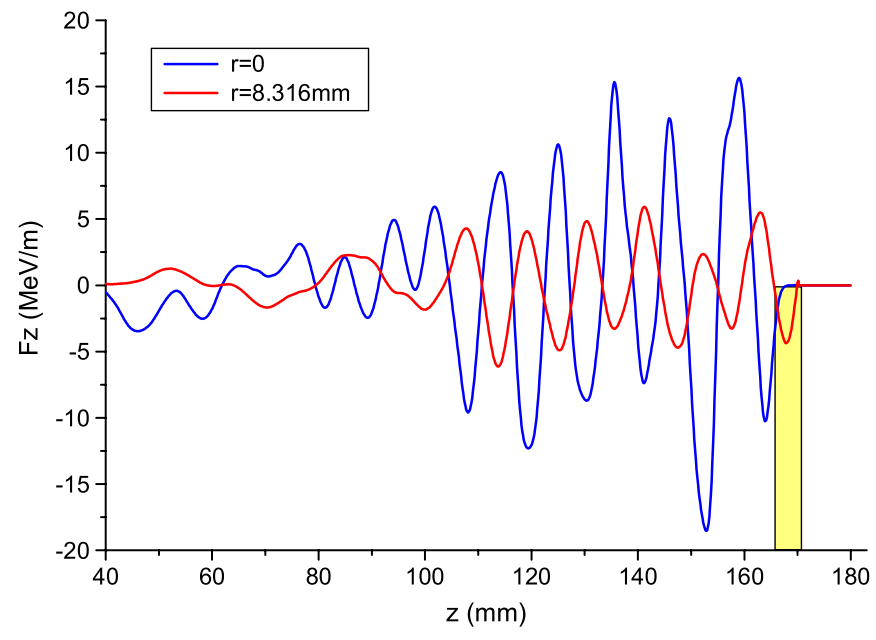

FIG. 2. Axial force from wakefields set up by a single $50 \mathrm{nC}$, $14 \mathrm{MeV}$ drive bunch in the CDWA structure described in Table I. The head of the drive bunch is located at $z=171 \mathrm{~mm}$ and the bunch travels from left to right.

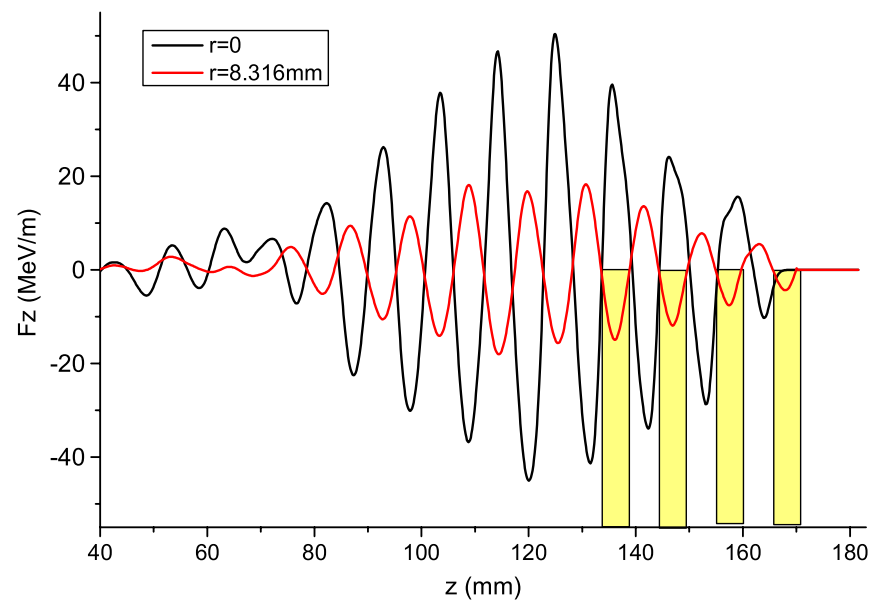

FIG. 3. Axial wakefields set up by four equally charged $50 \mathrm{nC}$, $14 \mathrm{MeV}$ drive bunches.

on the length of the structure and the group velocity of the waves set up by the passage of the drive bunch into the structure $[1,15,16]$.

We now set up a train of drive bunches. Figure 3 displays the wakefields set up by four equally charged $50 \mathrm{nC}$ drive bunches, spaced apart by the wakefield wavelength of the principal mode (10.66 $\mathrm{mm}$ ). The $\mathbf{T}$ calculated from the last drive bunch is 3.4 , essentially unchanged from the single bunch case. This choice achieves the maximum accelerating gradient $50 \mathrm{MeV} / \mathrm{m}$ for the witness bunch, which in this example can be located at $z=125 \mathrm{~mm}$. This choice of bunch train does not improve $\mathbf{T}$.

In Fig. 4, we change the distribution of charge among the bunches so that the ratios of bunch charge increases [7] from the first to the last bunch as 1:3:5:7. The $\mathbf{T}$ is 3.3. The conclusion thus far is that a train of multiple bunches having this variable charge, but with constant spacing of one wakefield period, does not improve $\mathbf{T}$. In this and the

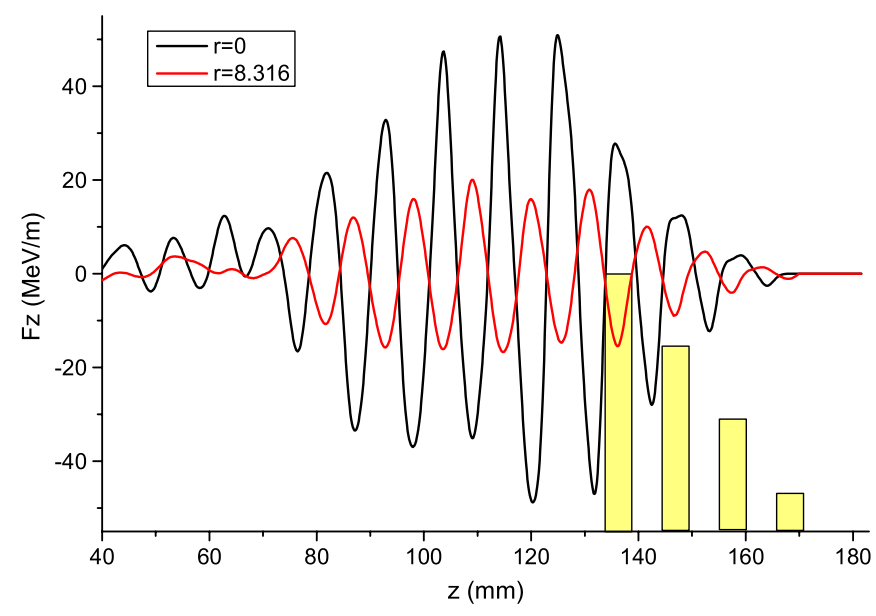

FIG. 4. Same as for Fig. 3, but now the bunch charge is increased as the ratio of odd numbers. 


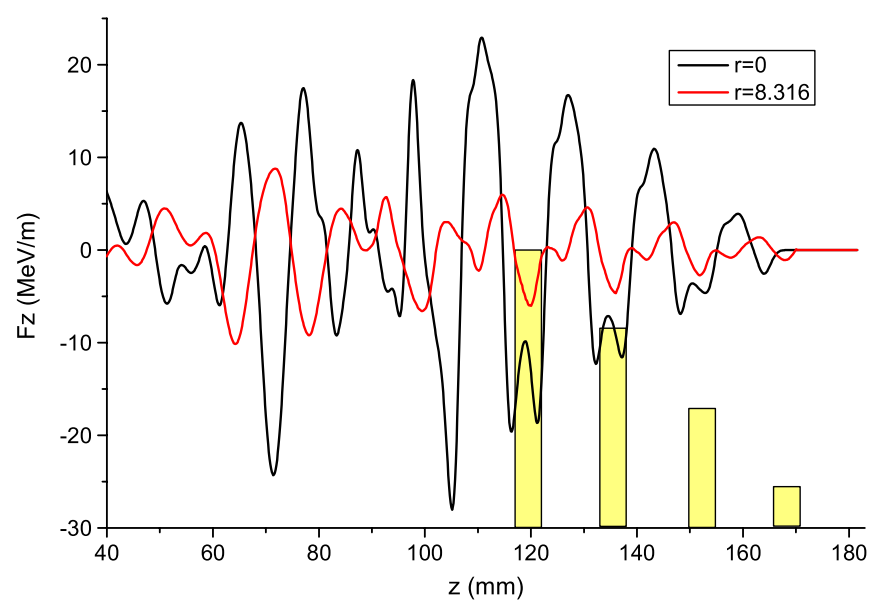

FIG. 5. Axial wakefields excited by a ramped bunch charge of drive bunches spaced by 1.5 wakefield wavelengths.

following examples, the total bunch charge for the four drive bunches is fixed at $200 \mathrm{nC}$.

In Fig. 5, the spacing of the bunches is changed to be one and one-half wakefield periods [7,17], but the bunch charge ratio is the same as for Fig. 4. The axial wakefield force is reduced, but that is to be expected, as this arrangement no longer provides maximum decelerating force for each bunch. The $\mathbf{T}$ is now 3.8: thus, the suggested algorithm of bunch charge ramping and spacing $[7,17]$ is not generally helpful [18], most likely because of the multimode behavior of the wakefields excited by this choice of drive bunches.

We next determine if there is some other choice of drive bunch charges and spacings that will improve $\mathbf{T}$, but yet result in a potentially sizable wakefield amplitude. To obtain an improved transformer ratio we proceed from the following, which is strictly proved for the collinear single-mode device: wakefields with maximum transformer ratio are generated by drive bunches whose particles lose the same energy [11,17]. From this statement it follows that for a train of point bunches with repetition period equal to a half wavelength (or one and a half wavelengths), to obtain the maximum transformer ratio the bunch charge should rise from head to tail in the train as the ratios of odd integer numbers. For the Gaussian longitudinal density of a bunch, the charge of the $n$th bunch should change according to the relation [17] $Q_{n}=Q_{1}[1+$ $T(n-1)]$, where $Q_{1}$ is the charge of the first bunch, and $\mathbf{T}$ is the transformer ratio of a single bunch. In the case of a multimode device, such a simple formula does not exist; therefore to obtain desirable locations of bunches and values of their charges it is necessary to run simulations, the number of which is equal to the number of bunches in the train. First, a calculation with one bunch is carried out, a location of the maximum of an accelerating field in the drive bunch channel is determined, and at this location the second bunch is placed and a new calculation of structure excitation is performed. At the location of the second

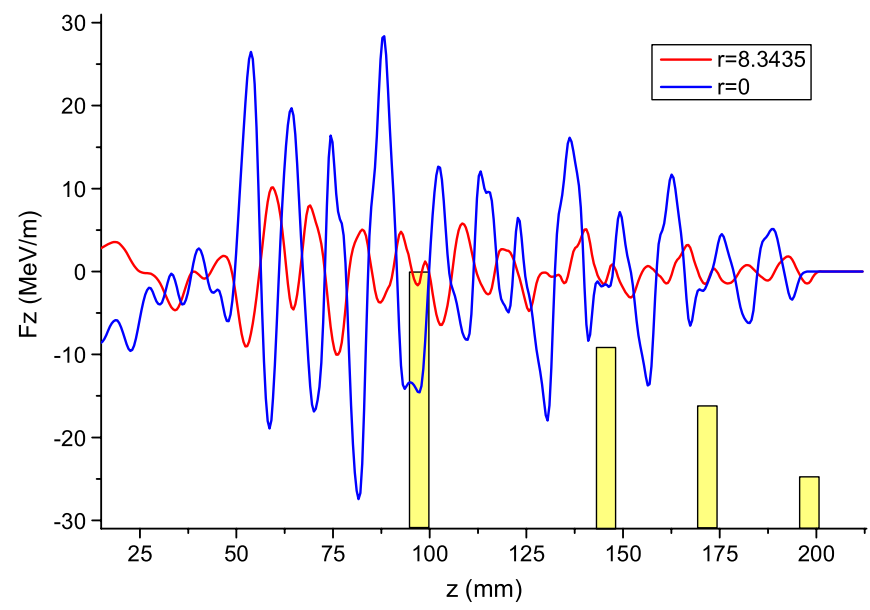

FIG. 6. In this example the bunch charges and spacings vary so as to enhance $\mathbf{T}$.

bunch, the superposition is such that the field there becomes decelerating. Then, each subsequent drive bunch is to be located at the maximum of the accelerating field from the prior bunches, and the charge of the $n$th bunch is set according to the formula $Q_{n}=Q_{1}\left[1+T_{n-1}\right]$, where $T_{n-1}$ is the transformer ratio after the $(n-1)$ st bunch. An answer to the goal of improved transformer ratio is provided by the result shown in Fig. 6. In this example the computation is run out to a greater distance, $201 \mathrm{~mm}$ to the front edge of the first drive bunch. The spaces between the bunches are, respectively, approximately 2.5 , 2.5 , and 4.5 wakefield periods, and the charge ratios are 1.0 , 2.4, 3.5, and 5.0; again, maintaining the constant total drive bunch charge at $200 \mathrm{nC}$. The $\mathbf{T}$ from the last bunch is 17 , a factor of 5 times as large as the $\mathbf{T}$ for the simple four-bunch train of equal charges spaced by one wakefield period. Notice the peak wakefield amplitude following the fourth drive bunch is only $28 \mathrm{MeV} / \mathrm{m}$, down from $50 \mathrm{MeV} / \mathrm{m}$ in

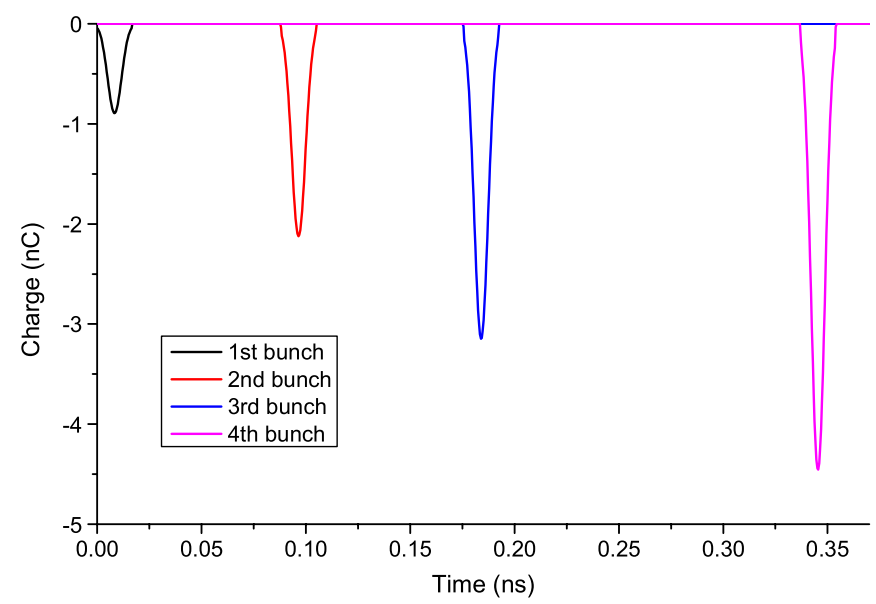

FIG. 7. Spacing and charge, emitted per one computation time step $\left(\Delta t=4.4 \times 10^{-4} \mathrm{~ns}\right)$, of the four drive bunches used for the computation of wakefields shown in Fig. 6. 
Figs. 3 and 4. This prescription for the drive bunch charge and spacing was obtained by striving for uniform drive bunch deceleration, which means that this arrangement should extract more energy from the entire drive bunch train than that, e.g., shown in Fig. 3. A graph of the four drive bunches, showing their relative charges, Gaussian shape, and spacing, is provided in Fig. 7. This example establishes that a considerable enhancement of $\mathbf{T}$ is possible in the multimode CDWA system using a flexible RBT method. The more complex algorithm (in comparison with $[7,17])$ for the bunch charge distribution and bunch locations is related to the multimode excitation of the CDWA. Of course, for single-mode structures, the conventional algorithm applies.

The nonuniform spacing of these drive bunches may present a problem for the accelerator system used to prepare them. However, the first three bunches are uniformly spaced, and the transformer ratio of the second bunch is 7.9, and after the third bunch it is 10.6. Thus, an experimental test of this method is feasible. The four drive bunches in the example of Fig. 6 each experience nearly the same decelerating force $(\sim 1.5 \mathrm{MeV} / \mathrm{m})$.

A natural question arises about the robustness of this method of optimization of the transformer ratio, namely, its sensitivity to fluctuations in charge distribution between the bunches or changes in the distances between the bunches. To answer this question, we performed a series of computations in which the ratio of the last bunch charge $Q_{4}$ to the first bunch charge $Q_{1}$ was varied, while the other parameters of the bunch train, including total charge, were fixed. The results of computations are shown in Fig. 8. A maximum transformer ratio of 18.6 is achieved for the charge ratio $Q_{4} / Q_{1}=4.8$. Decreasing the optimal charge ratio by $10 \%$, the transformer ratio falls to a value of 17 . Increasing the optimal charge ratio by $10 \%$, transformer ratio falls to a value 13.8. Thus, we can conclude that $20 \%$

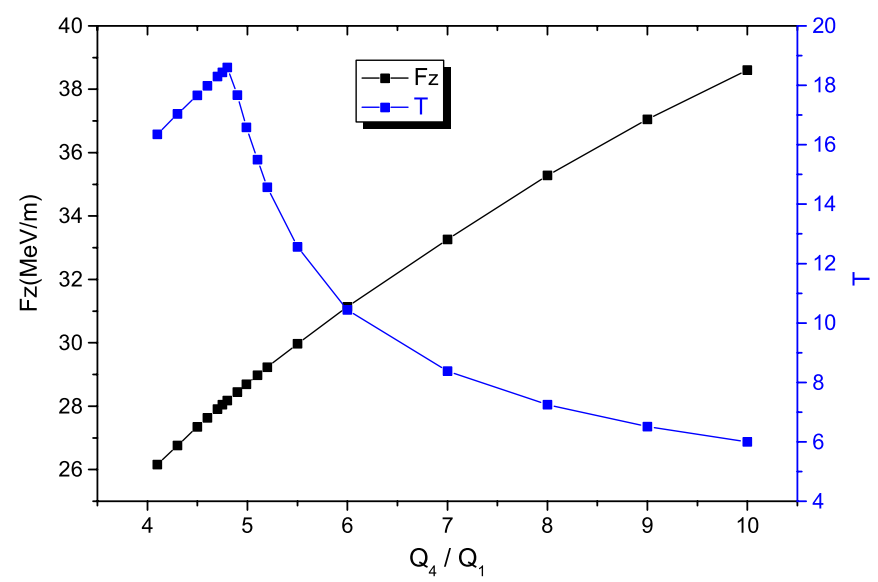

FIG. 8. Transformer ratio (blue symbols) and accelerating gradient (black symbols) versus the ratio of the last bunch charge to the first bunch charge. The other parameters of the bunch train and the CDWA are the same as in Fig. 6. uncertainty in the RBT charge distribution does not cause a catastrophic change of the transformer ratio. From these computations we find also the accelerating gradient is even less sensitive to the charge fluctuations. For the same 20\% range of the charge variation the value of the accelerating gradient changes from 26.7 to $29.5 \mathrm{MeV} / \mathrm{m}$.

Finally, we have studied the applicability of the ramped drive bunch train for four bunches to a high gradient $\mathrm{THz}$ CDWA structure [19]. Here the question is whether the removal of wakefields due to the group velocities phenomena will permit the superposition of wakefields from the train of delayed drive bunches, because the $\mathrm{THz}$ structure is very lightly loaded with dielectric and so the wave group velocity is very close to the particle velocity. It turns out that indeed all four drive bunches do not completely outrun the back front of the Cherenkov radiation in the short distance that we can study here. However, the back front of the wavefields does drop back significantly from each bunch of the train, enough so that one may locate the following bunch so as to engage the desirable effect of wakefield superposition and also find an enhanced $\mathbf{T}$. Thus, the RBT technique could find application in small $\mathrm{THz}$ collider-type structures. An example of this is shown in Fig. 9 for a diamond dielectric CDWA structure that has a radius $\sim 0.8 \mathrm{~mm}$ and a design frequency of $0.44 \mathrm{THz}$, where it can be seen that the wakefield amplitude builds up progressively behind the four drive bunches. For comparison, the four $5 \mathrm{GeV}$ drive bunches with charges totaling $6 \mathrm{nC}$ here set up a peak longitudinal wakefield amplitude $\sim 300 \mathrm{MeV} / \mathrm{m}$ with $T=17$, which is to be compared with a wakefield $\sim 500 \mathrm{MeV} / \mathrm{m}$ with $T=5.5$ from a single $6 \mathrm{nC}$ bunch [1].

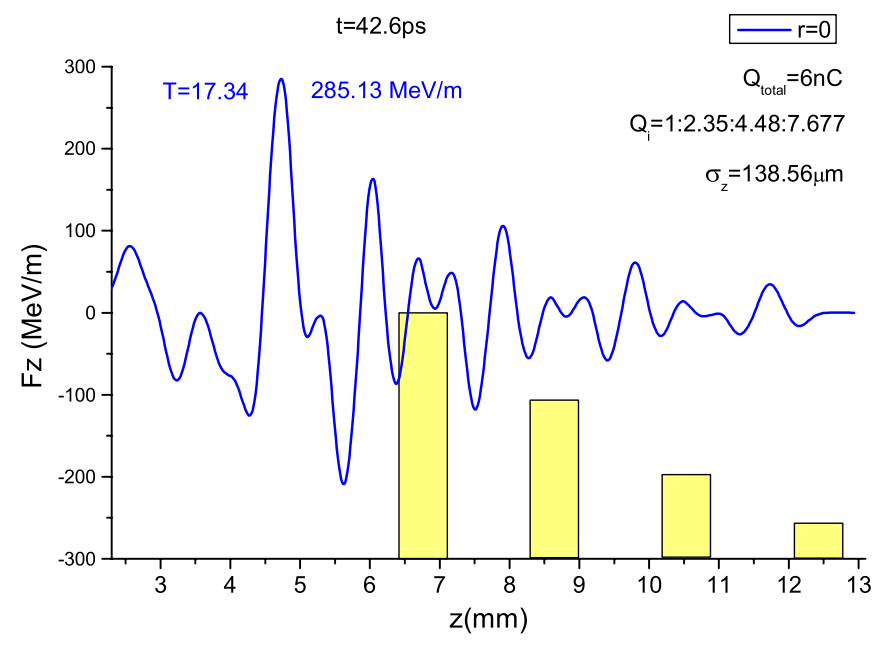

FIG. 9. Wakefield amplitude buildup from a four-drive bunch RBT in a THz CDWA. The bunch energy is $5 \mathrm{GeV}$, and the total charge $Q$, bunch rms size $\sigma_{z}, \mathbf{T}$, and charge ratios are displayed on the graph. The head of the first drive bunch is located at $z=12771 \mu \mathrm{m}$. The bunch spacing is approximately $1890 \mu \mathrm{m}$ and the bunch length parameter is $\sigma_{z}=139 \mu \mathrm{m}$. The bunches move from left to right. 


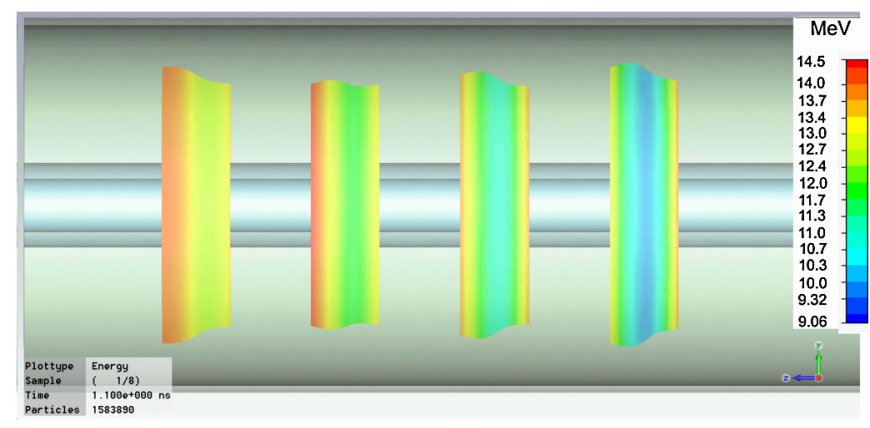

FIG. 10. Right side view of four drive bunches, initially centered on the structure axis, moving from right to left, along a CDWA structure specified in Table I. The color scale on the right shows the energy of the particles. These drive bunches are spaced apart by one period of the design mode, $10.67 \mathrm{~mm}$ and have moved 1.1 nsec.

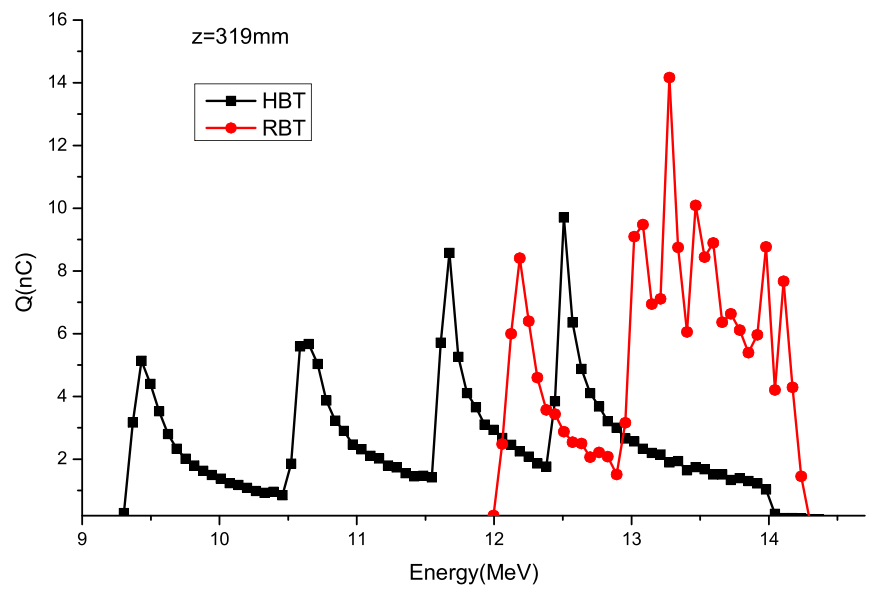

FIG. 11. Distribution functions of drive bunch particles after traveling $319 \mathrm{~mm}$ for the HBT (black symbols) and for the RBT (red symbols). The leftmost peak of the distribution function of the RBT is determined by the fourth bunch.

\section{MOTION OF DRIVE BUNCH TRAINS}

We begin by studying the motion of four drive bunches along the CDWA; the PIC code was run $1.1 \mathrm{nsec}(33 \mathrm{~cm})$ as the four drive bunches move along the structure, as specified in Table I. The drive bunches have equal charge and they are equally spaced by the period of principal wakefield mode. For this case, axial profiles of the wakefield were presented in Fig. 3. Figure 10 shows that at the end of travel, the drive bunches have moved with only slight distortion, which on further study is found to be a slight expansion/contraction of bunch diameter, symmetrically patterned around the azimuthal direction. Furthermore, it has been found displacement of the bunch by $1 \mathrm{~mm}$ from the axis of symmetry does not set up deflecting motions of the drive bunch train in this distance either [1]. Computational limitations preclude following the drive bunch train further, but this result establishes that, at least for this geometry, the motion of the drive bunches is approximately stable. Study of multi-GeV RBT stability for an accelerator having lengthy sections remains to be done.

From the energy scale given at the right side of Fig. 10 it follows that the maximum energy loss of bunch particles is $\sim 5 \mathrm{MeV}$; this coincides with estimates obtained from the maximum decelerating force $(\sim 15 \mathrm{MeV} / \mathrm{m})$ acting on the fourth drive bunch (see Fig. 3). A more precise value of 4.7 $\mathrm{MeV}$ and more detailed information about the energy distribution of all particles in the homogeneous bunch train (HBT) case of Fig. 3 is presented in Fig. 11 (black symbols and line). The four peaks on the distribution function correspond to the four drive bunches located in the linearly increasing retarding field. The average energy loss of all particles is $2.36 \mathrm{MeV}$. For comparison, in this figure is shown in red the energy distribution function of the ramped bunch train (Fig. 6) case. The average energy loss for the RBT is $0.76 \mathrm{MeV}$. Therefore, the travel distance of the RBT train should be greater by 3.1 times. The accelerating gradient for the RBT case is smaller, by a factor $\sim 1.8$, than the acceleration gradient for the HBT case. From these numbers we find that the energy gain of a test bunch in the RBT case will be greater by a factor 1.7 than the corresponding energy gain for the HBT. It should be noted that this number can be improved if we can reduce the unexpected energy loss of fourth bunch.

We now compare the results of Figs. 10 and 11 with a similar study for a cylindrical collinear DWA structure, specified in Table II. Essentially the structure has the same radius and outer alumina cylindrical shell as our CDWA

TABLE II. Parameters used for study of a cylindrical dielectric-lined collinear DWA.

\begin{tabular}{ll}
\hline \hline Design mode & $\sim 23.7 \mathrm{GHz}$ \\
External radius of dielectric tube & $14.05 \mathrm{~mm}$ \\
Inner radius of dielectric tube & $13.512 \mathrm{~mm}$ \\
Relative dielectric constant $\varepsilon$ & 9.8 \\
Bunch axial rms dimension $2 \sigma_{z}$ (Gaussian charge distribution) & $2.0 \mathrm{~mm}$ \\
Full bunch length used in PIC simulation & $5 \mathrm{~mm}$ \\
Drive bunch radius (box charge distribution) & $5 \mathrm{~mm}$ \\
Bunch energy & $14 \mathrm{MeV}$ \\
Total bunch charge & $200 \mathrm{nC}$ \\
Number of bunches & 4 \\
\hline \hline
\end{tabular}




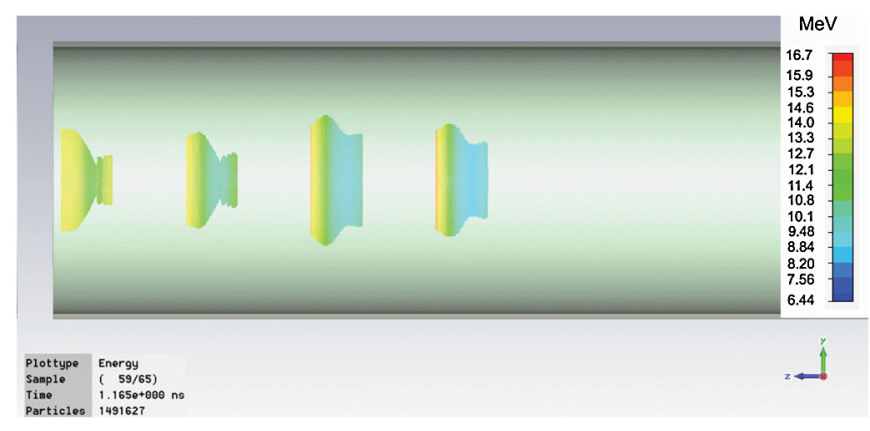

FIG. 12. Right side view of four drive bunches, initially centered on the structure axis, moving from right to left, along a cylindrical collinear DWA structure specified in Table II. The color scale on the right shows the energy of the particles. These drive bunches are spaced apart by one period of the design mode and are shown after 1.16 nsec of travel.

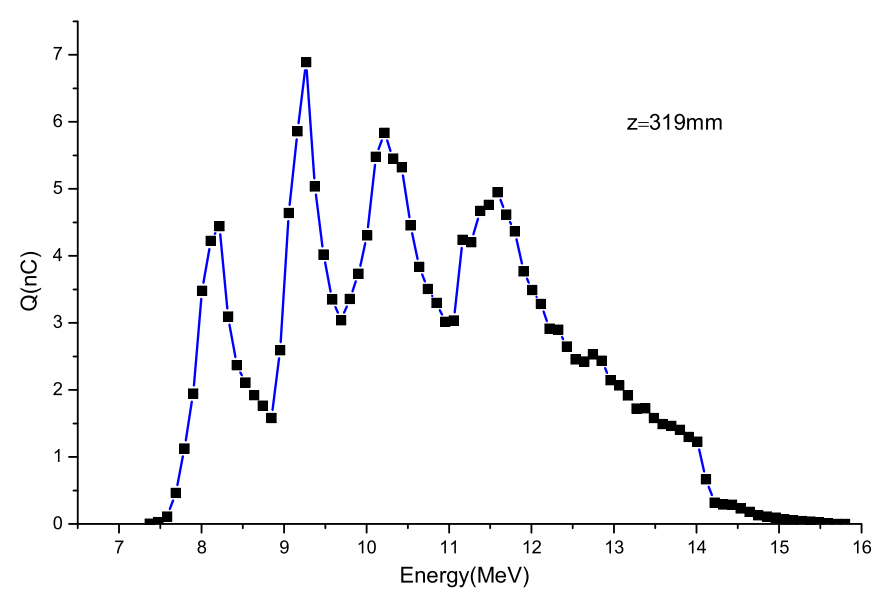

FIG. 13. Distribution function of HBT particles after traveling $319 \mathrm{~mm}$ in the cylindrical collinear DWA structure.

structure, but the central coaxial dielectric cylinder has been removed and the drive bunches are now cylindrical "pills" having radius $5 \mathrm{~mm}$ that have the same charge and energy as the annular drive bunches in Table I. Drive bunches are spaced apart by the wakefield wavelength of the principal mode $(12.64 \mathrm{~mm})$. Figure 12 shows the drive bunches at $1.16 \mathrm{nsec}$. It is apparent that the distortion of the drive bunches in the cylindrical collinear structure is more severe than in the CDWA structure. The field solutions in the cylinder are not the same as in the CDWA, so this result should not be surprising. These computations show that one must be cautious in assuming that the drive bunches move without significant changes in form. However, for purposes of this study of the CDWA, we believe it is fair to state that the drive bunches evidence minor modification of shape in the $20 \mathrm{~cm}(0.67 \mathrm{nsec})$ of travel that we used in the examples used in Sec. II.

The distribution function for the homogeneous bunch train for the cylindrical collinear DWA structure is shown in Fig. 13. Comparing this plot with the similar plot in
Fig. 11, one concludes that the energy spread of the drive bunch particles in the CDWA device is significantly less than in the collinear cylindrical DWA device. The average particle energy loss at the bunch location $z=319$ for the cylindrical collinear device is $3.36 \mathrm{MeV}$, i.e., greater by $\sim 1.5$ times than in the coaxial device, although the accelerating gradient after the bunch train is almost the same. Thus, the CDWA appears to be the preferred device for using the RBT technique.

\section{DISCUSSION}

By choosing a flexible algorithm for the charges and spacings of a short train of drive bunches that excite wakefields in a multimode dielectric wakefield accelerator, we have found that a considerable increase of the transformer ratio can be obtained. Furthermore, the use of such a programmed train of drive bunches permits the designer to control the transformer ratio independently of the dielectric structure parameters. The procedure for obtaining the best transformer ratio relies on the principle of having all drive bunches decelerate uniformly, but the use of numerical methods to obtain the result permits the designer to adapt the principle to the details of wakefield generation by the drive bunch train, such as the excitation of more than one wakefield mode. The method appears to be suitable for handling smaller structures that can generate very high acceleration gradients. The concept described here is limited to instantaneous excitation of a nonresonant (smooth) dielectric-lined waveguide by a train of only a few bunches.

While the motion of the bunch train could be studied numerically for $14 \mathrm{MeV}$ bunches traveling only $33 \mathrm{~cm}$, in this distance no breakup or deflection of the bunch train was found for the CDWA. An instability would be expected to affect the last drive bunch more than the first, which was not found to happen.

\section{ACKNOWLEDGMENTS}

This research was supported by the U.S. Department of Energy, Office of High Energy Physics, Advanced Accelerator R\&D. The authors thank Professor J.L. Hirshfield for his advice concerning this work.

[1] G. V. Sotnikov, T. C. Marshall, and J. L. Hirshfield, Phys. Rev. ST Accel. Beams 12, 061302 (2009).

[2] M.C. Thompson et al., Phys. Rev. Lett. 100, 214801 (2008).

[3] R. D. Ruth, A. W. Chao, P.L. Morton, and P. B. Wilson, Part. Accel. 17, 171 (1985).

[4] A. W. Chao, Physics of Collective Beam Instabilities in High Energy Accelerators (Wiley, New York, 1993).

[5] G. A. Voss and T. Weiland, Report No. DESY M-62-10, 1982. 
[6] K. L. F. Bane, P. B. Wilson, and T. Weiland, in Physics of High Energy Particle Accelerator, AIP Conf. Proc. No. 127 (AIP, New York, 1985), p. 875.

[7] C. Jing, A. Kanareykin, J. G. Power, M. Conde, Z. Yusof, P. Schoessow, and W. Gai, Phys. Rev. Lett. 98, 144801 (2007).

[8] C. Jing, J. G. Power, M. Conde, W. Liu, Z. Yusof, A. Kanareykin, and W. Gai, AAC: Fourteenth Workshop, edited by S. Gold and G. Nusinovich, AIP Conf. Proc. No. 1299 (AIP, New York, 2010), p. 348.

[9] K. L. Bane, P. Chen, and P. B. Wilson, IEEE Trans. Nucl. Sci. 32, 3524 (1985).

[10] J. T. Seeman, IEEE Trans. Nucl. Sci. 30, 3180 (1983).

[11] P. Schutt, T. Weiland, and V. M. Tsakanov, Probl. At. Sci. Technol., Ser. Nucl. Phys. Invest. 7, 12 (1990).

[12] J.P. Delahaye, CERN Particle Physics Seminar, http://clic-study.web.cern.ch/CLIC-Study/Presentations/ 20050421.pdf.

[13] E. Chojnacki, W. Gai, P. Schoessow, and J. Simpson, in Proceedings of the IEEE 1991 Particle Accelerator
Conference (APS Beams Physics) (IEEE, Piscataway, NJ, 1991), Vol. 5, pp. 2557-2559.

[14] M. E. Conde, W. Gai, R. Konecny, J. Power, P. Schoessow, and P. Zou, in AAC: Eighth Workshop, edited by W. Lawson, C. Bellamy, and D. F. Brosius, AIP Conf. Proc. No. 472 (AIP, New York, 1998), p. 626.

[15] I. N. Onishchenko, D. Yu. Sidorenko, and G. V. Sotnikov, Phys. Rev. E 65, 066501 (2002).

[16] V. A. Balakirev, I. N. Onishchenko, D. Yu. Sidorenko, and G. V. Sotnikov, J. Exp. Theor. Phys. 93, 33 (2001).

[17] S. S. Vaganyan, E. M. Lasiev, and V. M. Tsakanov, Probl. At. Sci. Technol., Ser. Nucl. Phys. Invest. 7, 32 (1990) (in Russian).

[18] Algorithm used in [7,17] for the increasing of the transformer ratio is correct, but its applicability is limited by single-mode structures.

[19] T. C. Marshall, G. V. Sotnikov, and J. L. Hirshfield, AAC: Fourteenth Workshop, edited by S. Gold and G. Nusinovich, AIP Conf. Proc. No. 1299 (AIP, New York, 2010), p. 336. 